\title{
OUTPUT FEEDBACK TRACKING SLIDING MODE CONTROL WITH PARALLEL FEEDFORWARD COMPENSATOR
}

\author{
Hirofumi Ohtsuka* Zenta Iwai ${ }^{* *}$ Ikuro Mizumoto ${ }^{* *}$ \\ * Kumamoto National College of Technology, Japan \\ ** Kumamoto University, Japan
}

\begin{abstract}
A new design method of output feedback type tracking sliding mode control (SMC) is proposed in this paper. Stability of the control system and the tracking performance to the reference input are achieved by using the parallel feedforward compensator ( $\mathrm{PFC}$ ), which guarantees almost strict positive realness (ASPR) of the plant, and the so-called internal model. Effectiveness of the method is shown through an experimental liquid-level control of the process. Copyright (C)2005 IFAC
\end{abstract}

Keywords: sliding mode control, tracking, output feedback, feedforward compensation, integral action, process control

\section{INTRODUCTION}

The sliding mode control (SMC) scheme has been applied to many industrial fields because of its superiority concerning robust control performance. From the viewpoint of its possibility of practical realization, SMC is expected to become more widely applicable control techniques. The design procedure of SMC system is divided into two stages. The first phase is to choose a set of switching surfaces such that the original system is restricted to the intersection of the switching surfaces. The second phase is to determine a switched control law that forces the system's trajectory to and maintains it on the sliding surface (V.I.Utkin, 1978). Unfortunately, most conventional methods in SMC employ either full state or estimated state feedback so that they may be impractical or overly complicated to implement. From the output feedback viewpoint, there have been several proposals concerning static output feedback and dynamic output feedback. For linear systems with no uncertainty, the problem of switching surface design using output information has been investigated (B.Diong and J.Medanic, 1992) . For uncer- tain systems, an algorithm for output-dependent switching surface design, which is based upon eigenvector methods, has been discussed by Hui and Zak (S.Hui and S.Zak, 1993) and Zak and Hui (S.Zak and S.Hui, 1993). Bag et al. (1997) indicated that the switching surface design problem can be reduced to an output feedback problem and gave the necessary and sufficient condition in terms of the system structure for a stable reduced order motion to exist. A common design methodology appears in the work of Heck et al. (1995). Elkhazali et al.(1995) is based on synthesizing a static output feedback gain numerically to ensure the so-called reachability condition. As an extension of the work by Edwards and Spurgeon (1995), a sliding mode controller synthesis procedure based on a linear matrix inequality (LMI) optimization has been proposed (C.Edwards and S.K.Spurgeon, 2001). On the contrary, Ohtsuka et al. (2003) have proposed a different design procedure by using a parallel feedforward compensator (PFC). The design method is based on the almost strict positive realness (ASPR) of the plant and the PFC is utilized to realize the ASPR of the 
minimal phase plant. The above stated idea was originally used in the design of simple adaptive (SAC) systems (Z.Iwai and I.Mizumoto, 1994). In this case, the sliding mode switching surface can be specified by an augmented plant output with PFC which guarantees the almost strict positive real characteristics of the augmented plant. This approach was also adopted by Ohtsuka et al.(2004) to construct the output feedback servo control for practical electromechanical systems.

In this report, a new control procedure is proposed which is an extension of the work of Ohtsuka et al. (2004) and is based on an internal model principle. The method has the following attractive features: 1) The design technique is easy for designing the servo control system and the obtained controller structure is simple. 2) The SMC can be realized without time derivative of the plant output signal. 3) The chattering phenomenon can be suppressed by introducing an integral action.

This paper is organized as follows. Section 2 presents the problem formulation. Section 3 describes the scheme of a new controller and discusses the stability of the proposed SMC system. Section 4 shows a concrete and practical control system construction method using PFC. An experimental result is included in Section 5 to illustrate the design procedure and to demonstrate the effectiveness of the proposed approach.

\section{PROBLEM FORMULATION}

Consider the following controllable and observable SISO linear system:

$$
\begin{aligned}
& \dot{x}(t)=A x(t)+b u(t), \\
& y(t)=c^{\mathrm{T}} x(t),
\end{aligned}
$$

where $x \in R^{n}, A \in R^{n \times n}, b, c \in R^{n \times 1}$. The problem to be discussed is a construction of a sliding mode control system which can achieve the tracking of output $y(t)$ to the reference input $r(t)$ by output feedback type. Suppose that $r(t)$ satisfies the following differential equation which is known as an internal model:

$$
\begin{gathered}
D_{a}(s) r(t)=0, \\
D_{a}(s)=s^{\rho}+d_{1} s^{\rho-1}+\cdots+d_{\rho} .
\end{gathered}
$$

Here $s$ denotes the differential operator.

Define $z(t), v(t)$ and the tracking error $e(t)$ as

$$
\begin{aligned}
z(t) & =D_{a}(s) x(t), \\
v(t) & =D_{a}(s) u(t), \\
e(t) & =y(t)-r(t), \\
z(t) \in R^{n} & , v(t) \in R^{1}, e(t) \in R^{1} .
\end{aligned}
$$

Then operating $D_{a}(s)$ for both sides of $(7)$ and considering (3) lead to the following equation:

$$
D_{a}(s) e(t)=c^{T} z(t),
$$

or

$$
e^{(\rho)}+d_{1} e^{(\rho-1)}+\cdots+d_{\rho} e^{(0)}=c^{T} z(t),
$$

where $e^{(0)}=e(t)$ and $e^{(i)}$ denotes the $i$-th time derivative of $e(t)(i=0 \cdots \rho)$. From $(1),(5),(6)$ and (9), we can obtain the following equation:

$$
\begin{aligned}
& {\left[\begin{array}{c}
\dot{z}(t) \\
e^{(1)} \\
\vdots \\
e^{(\rho)}
\end{array}\right]=\left[\begin{array}{cccc}
A & 0 & \cdots & 0 \\
0^{T} & 1 & \ddots & \vdots \\
\vdots & \ddots & \ddots & 0 \\
0^{T} & \cdots & 0 & 1 \\
c^{T} & -d_{\rho} & \cdots & -d_{1}
\end{array}\right]\left[\begin{array}{c}
z(t) \\
e^{(0)} \\
\vdots \\
e^{(\rho-1)}
\end{array}\right]+\left[\begin{array}{c}
b \\
0 \\
\vdots \\
0
\end{array}\right] v(t)} \\
& \sigma(t)=e(t)=e^{(0)} \\
& =\left[\begin{array}{lllll}
0^{T} & 1 & 0 & \cdots & 0
\end{array}\right]\left[\begin{array}{c}
z(t) \\
e^{(0)} \\
\vdots \\
e^{(\rho-1)}
\end{array}\right] .
\end{aligned}
$$

In the above system, the tracking error $e(t)$ and the extended input $v(t)$ are designated as the output and input, respectively. For brevity, the above equations are rewritten as follows:

$$
\begin{aligned}
\frac{d}{d t} \bar{x}(t) & =\bar{A} \bar{x}(t)+\bar{b} v(t), \\
\sigma(t) & =\bar{c}^{T} \bar{x}(t) .
\end{aligned}
$$

In this case, we have

$$
\bar{c}^{T} \bar{b}=0 .
$$

That is, the relative degree is equal or greater than 2 so that the output feedback control has no effect upon the system in (12) and (13). To improve the situation, we introduce the following parallel feedforward compensator(PFC):

$$
\begin{aligned}
\frac{d}{d t} x_{f}(t) & =A_{f} x_{f}(t)+b_{f} v(t), \\
y_{f}(t) & =c_{f}^{T} x_{f}(t),
\end{aligned}
$$

where $x_{f} \in R^{n_{f}}, A_{f} \in R^{n_{f} \times n_{f}}, b_{f}, c_{f} \in R^{n_{f} \times 1}$. Hence we have the following extended plant:

$$
\begin{aligned}
\frac{d}{d t} x_{a}(t) & =A_{a} x_{a}(t)+b_{a} v(t), \\
\sigma_{1}(t) & =\sigma(t)+y_{f}(t)=c_{a}^{T} x_{a}(t),
\end{aligned}
$$

where

$$
\begin{aligned}
& x_{a}=\left[\begin{array}{c}
\bar{x} \\
x_{f}
\end{array}\right], A_{a}=\left[\begin{array}{cc}
\bar{A} & 0 \\
0 & A_{f}
\end{array}\right], \\
& b_{a}=\left[\begin{array}{c}
\bar{b} \\
b_{f}
\end{array}\right], c_{a}=\left[\begin{array}{c}
\bar{c} \\
c_{f}
\end{array}\right] .
\end{aligned}
$$


Assumption 1

Extended plant (17) with output $\sigma_{1}(t)$ and input $v(t)$ is ASPR.

It is noted that the system in (17) and (18) is said to be ASPR if the corresponding transfer function:

$$
G_{a}(s)=c_{a}^{T}\left(s I-A_{a}\right)^{-1} b_{a}
$$

satisfies the following ASPR conditions (E.Zeheb, 1986):

(1) The relative degree is 0 or 1 .

(2) The leading coefficient is positive.

(3) The numerator of $G_{a}(s)$ is Hurwitz.

It follows that

$$
c_{a}^{T} b_{a}=c_{f} b_{f}>0
$$

by Assumption 1.

\section{SLIDING MODE CONTROL LAW AND STABILITY}

Let us consider the following control law:

$$
v(t)=-\frac{1}{c_{a}^{T} b_{a}}\left(c_{a}^{T} A_{a} x_{a}(t)+k \operatorname{sgn} \sigma_{1}(t)\right) .
$$

Here the relay gain $k=k\left(x_{a}, t\right) \leq k_{0}$ is a design parameter and $\operatorname{sgn} \sigma_{1}(t)$ is defined as the following function:

$$
\operatorname{sgn} \sigma_{1}(t)= \begin{cases}1 & \left(\sigma_{1}>0\right) \\ 0 & \left(\sigma_{1}=0\right) \\ -1 & \left(\sigma_{1}<0\right)\end{cases}
$$

where $k_{0}$ is an positive constant. The control law (21) is known as the ultimate sliding mode. Namely, the state trajectory of (17) is drawn to the switching hyper plane from an arbitrary initial point and also held on it once the state trajectory reaches to the surface.

\section{Theorem}

Under Assumption 1, the control law in (21) with relay element (22) attains the relation:

$$
\lim _{t \rightarrow \infty} e(t)=0
$$

Proof:

Let us consider the positive definite function

$$
V(t)=\frac{1}{2} \sigma_{1}(t)^{2}
$$

as a candidate Lyapnov function. Then

$$
\begin{aligned}
\frac{d}{d t} V(t) & =\sigma_{1}(t) \dot{\sigma}_{1}(t) \\
& =\sigma_{1}(t)\left(c_{a}^{T} A_{a} x_{a}(t)+c_{a}^{T} b_{a} v(t)\right) \\
& =\sigma_{1}(t)\left\{c_{a}^{T} A_{a} x_{a}(t)-c_{a}^{T} A_{a} x_{a}(t)\right.
\end{aligned}
$$

$$
\begin{gathered}
\left.-k \operatorname{sgn} \sigma_{1}(t)\right\} \\
=-k\left|\sigma_{1}(t)\right| \\
\leq-k_{0}\left|\sigma_{1}(t)\right|<0\left(\sigma_{1}(t) \neq 0\right) .
\end{gathered}
$$

Thus, the trajectory of state (17) reaches the sliding mode switching hyper plane $\sigma_{1}(t)=0$, that is

$$
\lim _{t \rightarrow \infty} \sigma_{1}(t)=0
$$

The behavior of the trajectory on the hyper plane $\sigma_{1}=0$ can be analyzed by the equivalent linear control method (J.A.Burton and A.S.I.Zinober, 1986). In this case, we have $\dot{\sigma}_{1}(t)=0$. Then, we have

$$
c_{a}^{T} A_{a} x_{a}(t)+c_{a}^{T} b_{a} v(t)=0
$$

Thus, the equivalent linear control input is given as

$$
v_{e q}(t)=-\frac{1}{c_{a}^{T} b_{a}} c_{a}^{T} A_{a} x_{a}(t)
$$

Substituting (28) into (17) yields

$$
\begin{aligned}
\dot{x}_{a}(t) & =\left(A_{a}-\frac{1}{c_{a}^{T} b_{a}} b_{a} c_{a}^{T} A_{a}\right) x_{a}(t) \\
& =\hat{A}_{a} x_{a}(t) .
\end{aligned}
$$

The eigenvalues of $\hat{A}_{a}$ consist of the $n-1$ zeros of the extended system in (17) and (18) and a single zero on the origin of the complex plane. Hence, according to Assumption 1, (29) is stable (O.M.E. El-Ghezawi and Zinober, 1983), that is,

$$
\lim _{t \rightarrow \infty} x_{a}(t)=0
$$

or

$$
\lim _{t \rightarrow \infty} e^{(0)}=\lim _{t \rightarrow \infty} e(t)=0
$$

\section{ON THE CONCRETE CONSTRUCTION OF CONTROL SYSTEM}

\subsection{Construction of the Control Input $u(t)$}

The term $c_{a}^{T} A_{a} x_{a}(t)$ in $(21)$ can be rewritten as follows:

$$
\begin{aligned}
c_{a}^{T} A_{a} x_{a}(t) & =\left[\begin{array}{ll}
\bar{c}^{T} & c_{f}^{T}
\end{array}\right]\left[\begin{array}{cc}
\bar{A} & 0 \\
0 & A_{f}
\end{array}\right]\left[\begin{array}{c}
\bar{x}(t) \\
x_{f}(t)
\end{array}\right] \\
& =\bar{c}^{T} \bar{A} \bar{x}(t)+c_{f}^{T} A_{f} x_{f}(t) \\
& =e^{(1)}+c_{f}^{T} A_{f} x_{f}(t) \\
& =\frac{d}{d t} e(t)+c_{f} A_{f} x_{f}(t) .
\end{aligned}
$$

From $c_{a}^{T} b_{a}=c_{f}^{T} b_{f}$ and (32), the control input in (21) is given by 


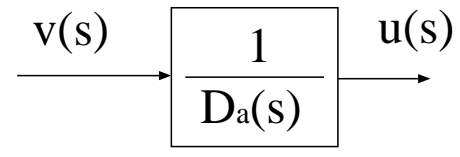

(a)

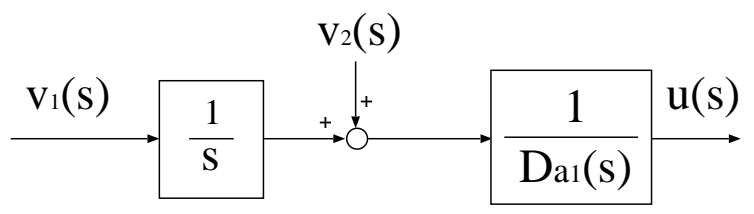

(b)

Fig.4.1 Reference model filter

$v(t)=-\frac{1}{c_{f}^{T} b_{f}}\left(\frac{d e(t)}{d t}+c_{f} A_{f} x_{f}(t)+k \operatorname{sgn} \sigma_{1}(t)\right)$.

It is emphasized that the input $v(t)$ can be constructed by measurable variables $y(t), r(t), y_{f}(t)$ and $x_{f}(t)$ and time derivative $\dot{y}_{f}(t)$. From $(6), u(t)$ can be described by

$$
u(t)=\frac{1}{D_{a}(s)} v(t) .
$$

It means that the reference model filter, represented by $1 / D_{a}(s)$, generates the real input $u(t)$ from $v(t)$.

In (33), $v(t)$ includes the time derivative $\dot{e}(t)$ or the time derivative $\dot{y}(t)$. If the latter signal can not be measured, it is often calculated from $y(t)$ by some approximation, e.g., the Euler method, or the differential circuit. However it commonly amplifies the influence of calculation errors and/or measurement noises. This situation can be avoided in case that $D_{a}(s)$ has the following form:

$$
\begin{aligned}
D_{a}(s) & =s D_{a 1}(s), \\
D_{a 1}(s) & =d_{1} s^{\rho-2}+\cdots+d_{\rho-1}, d_{\rho}=0 .
\end{aligned}
$$

Because, in this case, we have

$$
\begin{aligned}
v(t) & =v_{1}(t)+\dot{v}_{2}(t) \\
v_{1}(t) & =-\frac{1}{c_{f}^{T} b_{f}}\left(c_{f}^{T} A_{f} x_{f}(t)+k \operatorname{sgn} \sigma_{1}(t)\right) \\
v_{2}(t) & =-\frac{1}{c_{f}^{T} b_{f}} e(t)
\end{aligned}
$$

(see Fig 4.1(b)). It is noted that the chattering problem may also be improved by passing through $v_{1}(t)$ at least an integrator. Furthermore, the chattering problem can be improved more by using some conventional countermeasure as $\sigma$ modification (K.K.D.Young, 1993).

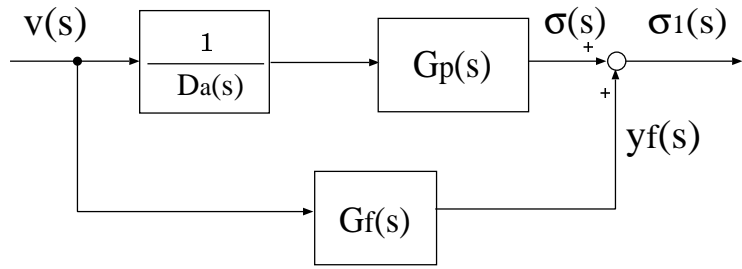

Fig.4.2 Augmented plant with PFC

\subsection{Construction of PFC}

From (5),(6) and (8), we have

$$
\sigma(s)=e(s)=\frac{1}{D_{a}(s)} G_{p}(s) v(s),
$$

where $\sigma(s), e(s)$, and $v(s)$ are Laplace transforms of $\sigma(t), e(t)$ and $v(t)$, respectively. $G_{p}(s)$ denotes the transfer function of the controlled plant in (1) and (2):

$$
G_{p}(s)=c^{T}(s I-A)^{-1} b .
$$

The PFC can be described by the transfer function form:

$$
G_{f}(s)=c_{f}^{T}\left(s I-A_{f}\right)^{-1} b_{f} .
$$

Hence, the ASPR of the augmented system,

$$
\begin{aligned}
G_{a}(s) & =G_{p 1}(s)+G_{f}(s), \\
G_{p 1}(s) & =\frac{1}{D_{a}(s)} G_{p}(s),
\end{aligned}
$$

must be kept to satisfy Assumption 1 (see Fig.4.2).

Let $G_{p}(s)$ be given as

$$
G_{p}(s)=\frac{k_{p} N_{p}(s)}{D_{p}(s)},
$$

where $D_{p}(s)$ and $N_{p}(s)$ are monic polynomials with $n$-th order and $m$-th order, respectively, and $k_{p}$ is a leading coefficient. Then,

$$
G_{p 1}(s)=\frac{k_{p} N_{p}(s)}{D_{a}(s) D_{p}(s)}
$$

can be regarded as an extended system involving the internal model of the reference input with relative degree $\gamma=n+\rho-m$. It is apparent that the zeros of $G_{p 1}(s)$ is determined by zeros of the originally controlled plant $G_{p}(s)$. Hence, under the assumptions that $k_{p}>0, \gamma$ is known and $N_{p}(s)$ is Hurwitz polynomial, the ladder networktype PFC (Fig.4.3), which has been proposed by Iwai et al. (Z.Iwai and M.Deng, 1994), is considerably useful to realize an ASPR augmented plant. In this case, $G_{f}(s)$ can be designed as follows: 


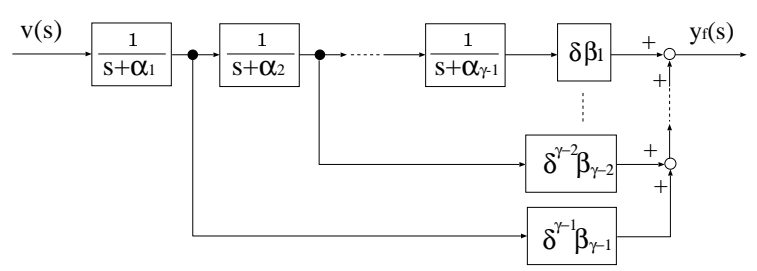

Fig.4.3 Concrete construction of PFC

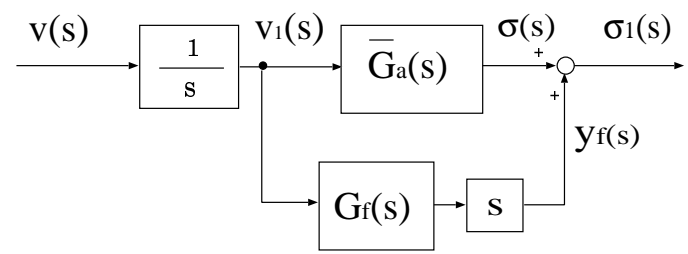

Fig.4.4 Modification of PFC

$$
\begin{aligned}
G_{f}(s)= & \sum_{i=1}^{\gamma-1} \delta^{i} G_{f i}(s) \\
G_{f i}(s)= & \frac{\beta_{i}}{d_{i}(s)} \\
d_{i}(s)= & \prod_{j=1}^{\gamma-1}\left(s+\alpha_{i}\right) \\
\delta: & \text { small positive constant } \\
\beta_{i}: & \text { coefficients of Hurwitz polynomial: } \\
& \beta_{\gamma-1} s^{\gamma-1}+\cdots+\beta_{1} s+k_{p}
\end{aligned}
$$

Then there exists a positive constant $\delta_{0}$ such that $G_{a}(s)$ becomes ASPR for $\delta$ as $\delta_{0}>\delta>0$.

\subsection{Modification of PFC in case of (35)}

The input of PFC, designed in the preceding subsection 4.2 , can be constructed by using the time derivative of $y(t)$ as mentioned in subsection 4.1. To avoid this situation, we introduce $\bar{v}(t)$ using (37) and (38) such that

$$
\bar{v}(t)=\int_{0}^{t} v(t) d t=\int_{0}^{t} v_{1}(t) d t+v_{2}(t) .
$$

However, in this case, we need to modify the form of PFC corresponding to the change of the input to PFC. Let

$$
\bar{G}_{a}(s)=\frac{1}{D_{a 1}(s)} G_{p}(s),
$$

where $D_{a 1}(s)$ is given in (35). In this case, PFC $G_{f}(s)$ is designed so as to make $G_{p 1}(s)=$ $\frac{1}{s} \bar{G}_{a}(s)$ as an ASPR system by the abovementioned scheme shown in subsection 4.2. Fig.4.4 describes the connection between $\bar{G}_{a}(s)$ and $G_{f}(s)$ by block diagrams. It can be easily transformed to an equivalent diagram in Fig. 4.2 by the shift of the feeding out point of the input for $G_{f}(s)$. Since the PFC has been described by (15) and (16), we need to reconstruct it as

$$
\begin{aligned}
& \dot{x}_{f}(t)=A_{f} x_{f}(t)+b_{f} \bar{v}(t), \\
& \bar{y}_{f}(t)=\dot{y}_{f}(t)=c_{f}^{T} A_{f} x_{f}(t),+c_{f}^{T} b_{f} \bar{v}(t)
\end{aligned}
$$

based on the above equivalent transformation of the block diagram in Fig.4.4. As a result, we can avoid using the time derivative of the plant output $y(t)$ and realize the output feedback type sliding mode control system in case of (35).

\section{EXPERIMENTAL RESULTS}

Here the proposed method is applied to a process control example in order to examine the concrete design procedure and to confirm the control performance. Fig.5.1 shows a schematic diagram of the experimental equipment with three tanks. The operational input is the volume of inflowing water of tank- 2 generated by pump- 1 and the controlled value is the water level of tank- 1 which is indirectly measured by a pressure sensor. The following transfer function:

$$
G_{p}(s)=\frac{13.29 s+0.6916}{s^{2}+1.654 \times 10^{-2} s+3.14 \times 10^{-5}}
$$

of the plant was obtained from the step response of the system by using the exponential analysis method based on Plony's method (Z.Iwai and I.Torigoe, 2003). The control objective is to realize the tracking of the output to the reference input $r(t)$ composed of the step signal and ramp signal.

\subsection{Design of the SMC System}

Since the reference input includes a ramp signal, $D_{a}(s)$ is chosen as $1 / s^{2}$. In this case, the relative degree of $G_{p 1}(s)$ becomes 3 . So, we chose the 2nd order PFC of which parameters are shown in table 5.1. The switching gain function $k\left(x_{a}, t\right)$ is

Table 1. PFC design parameters

\begin{tabular}{c|ccccc}
\hline symbol: & $\delta$ & $\alpha_{1}$ & $\alpha_{2}$ & $\beta_{1}$ & $\beta_{2}$ \\
\hline value: & 0.1 & $10^{-4}$ & $10^{-4}$ & $10^{3}$ & $10^{4}$ \\
\hline
\end{tabular}

chosen as $k\left(x_{a}, t\right)=k_{0}=10^{-6}$. Fig.5.2 shows an experimental result. We can see sufficiently good control performance: the switching function $\sigma(t)$ approaches zero and the output tracking of $y(t)$ to the reference input with both of ramp and step signals is also attained.

\section{CONCLUSION}

We have proposed a design scheme of output feedback sliding mode tracking controllers by using a parallel feedforward compensator which has been used in the design of simple adaptive control 


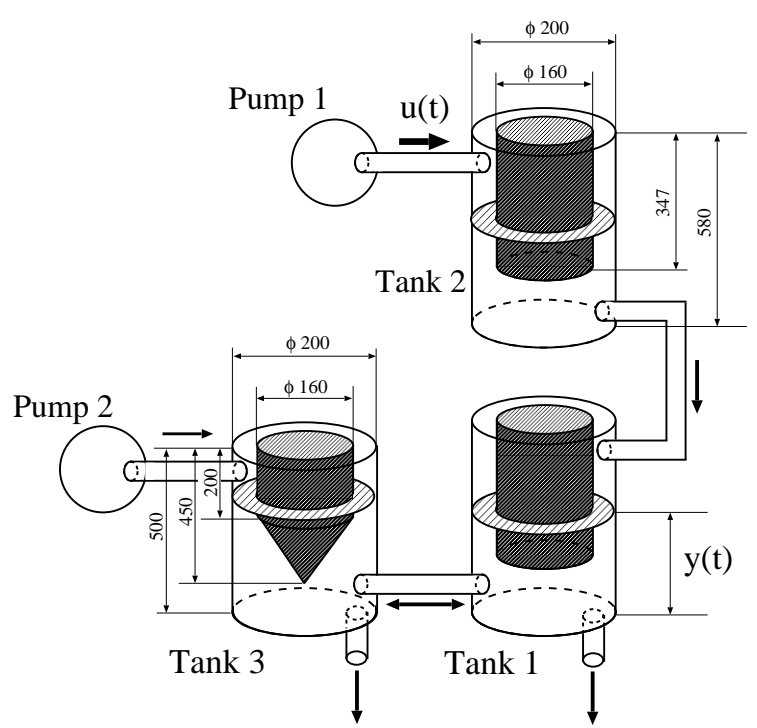

Fig.5.1 Schematic of tank system

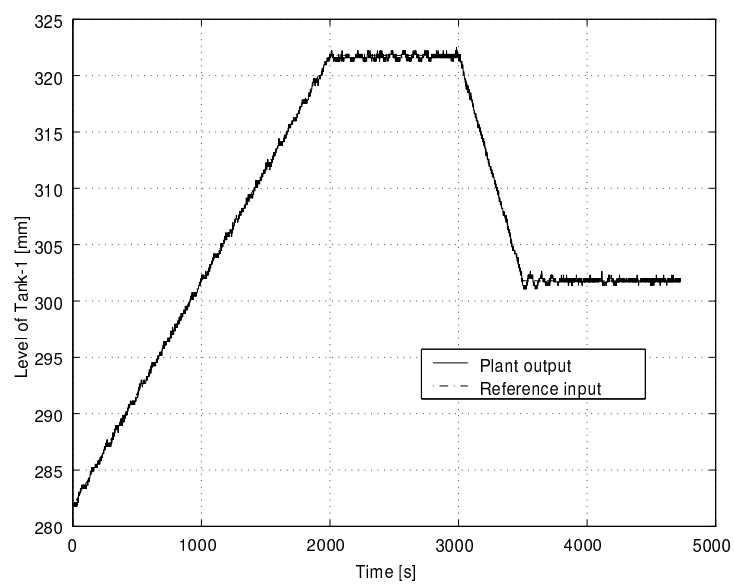

(a) Response of plant $y(t)$ and $r(t)$

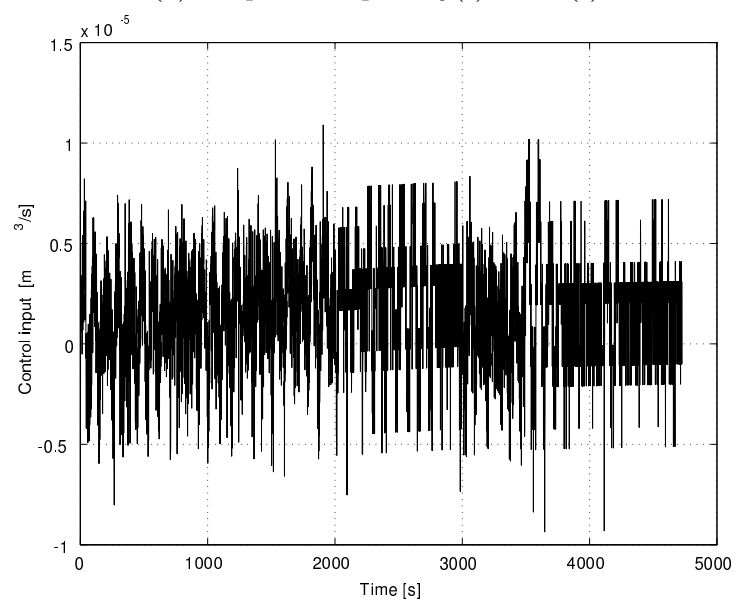

(b) Control input $u(t)$

Fig.5.2 Experimental result

systems. Stability of the control system is guaranteed under the assumption that the original plant is minimal phase and the relative order is known. Several design techniques are derived on the concrete construction of the controller. The effectiveness of the proposed method was confirmed through experimental results on a liquidlevel control process. In this experiment, the con- trolled system, which is consisted of the connected three tanks, was approximated as 2nd order transfer function based on the step type response, in spite of that it must be originally characterized as 3rd order non-linear differential equation from its structure. Therefore, it has to be emphasized that this experimental result shows the the robust-ness of the proposed output feedback type SMC system for structural uncertainties of the controlled plant.

\section{REFERENCES}

B.Diong and J.Medanic (1992). Dynamic output feedback variable structure control for system stabilisation. Int.J.Control 56, 607-630.

C.Edwards,

A.Akoachere and S.K.Spurgeon (2001). Sliding mode output feedback controller design using linear matrix inequalities. IEEE Trans. on Automatic Control 46-1, 115-119.

E.Zeheb (1986). A sufficient condition for output feedback stabilization of uncertain systems. IEEE Trans. on A.C. 31-11, 1055-1057.

J.A.Burton and A.S.I.Zinober (1986). Continuous approximation of variable structure control. Int.J.Sys., Sci. 6, 875-885.

K.K.D.Young (1993). Variable Structure Control for Robotics and Aerospace Applications. Elsvier.

O.M.E. El-Ghezawi, S.A. Billings and A.S.I. Zinober (1983). Variable-structure systems and system zeros. Proceedings of IEE, Part D 130-1, 1-5.

S.Hui and S.Zak (1993). Robust output feedback stabilisation of uncertain dynamic systems with bounded controllers. Int.J.of Robust and Nonlinear Control 3, 115-132.

S.Zak and S.Hui (1993). Output feedback in variable structure controllers and state estimators for uncertain/nonlinear dynamic systems. Proceedings of the IEE, Part D 140, 41-50.

V.I.Utkin (1978). Sliding Modes and Their Application in Variable Structure Systems. Mir. Moscow.

Z.Iwai and I.Mizumoto (1994). Realization of simple adaptive control by using parallel feedforward compensator. Int.J.Control 59-6, 15341565 .

Z.Iwai, I.Mizumoto and M.Deng (1994). A parallel feedforward compensator virtually realizing almost strictly positive real plan. Proceedings of the 33rd CDC, Florida, US pp. 2827-2832.

Z.Iwai, I.Mizumoto, M.Kumon and I.Torigoe (2003). Modelling of time delay systems using exponential analysis method. Proceedings of the International Conference of Cottrol, Automation and Systems, Korea pp. 2298-2303. 